\title{
A Scintillation Hodoscopic Spectrometer
}

\author{
V. I. Alekseev ${ }^{a}$, V. A. Baskov ${ }^{a, *}$, V. A. Dronov ${ }^{a}$, A. I. L'vov ${ }^{a}$, A. V. Koltsov ${ }^{a}$, \\ Yu. F. Krechetov ${ }^{b}$, V. V. Polyansky ${ }^{a}$, and S. S. Sidorin ${ }^{a}$ \\ ${ }^{a}$ Lebedev Physical Institute (LPI), Russian Academy of Sciences, \\ Moscow, 119991 Russia \\ ${ }^{b}$ Joint Institute for Nuclear Research (JINR), \\ Dubna, Moscow oblast, 141980 Russia \\ *e-mail: baskov@x4u.lebedev.ru \\ Received June 7, 2020; revised July 11, 2020; accepted July 29, 2020
}

\begin{abstract}
The calibration results are presented for a scintillation hodoscopic spectrometer of charged particles, which has been designed for an experiment on the search for a heavy electron on the bremsstrahlung photon beam at the Pakhra accelerator of the LPI. The relative energy and coordinate resolutions of the spectrometer at electron energy $E=40 \mathrm{MeV}$ are $\delta=22 \%$ and $\sigma_{x}=9.5 \mathrm{~mm}$, respectively. It has been determined that the width of electromagnetic showers in the transverse direction can be described by the energy dependences $\Delta \sim \ln E$ at $E<100 \mathrm{MeV}$ and $\Delta \sim 1 / \exp E$ at $E>100 \mathrm{MeV}$.
\end{abstract}

DOI: $10.1134 / \mathrm{S} 0020441221010024$

\section{INTRODUCTION}

The possible existence of light long-lived charged particles $\ell^{ \pm}$with a mass in the range between the electron and muon masses, which have not yet been observed, has been discussed theoretically and tested experimentally many times. All search results were negative; this is the reason that the appearance of information about the observation of such light particles with masses of approximately $9 \mathrm{MeV}$ on stereo photographs taken in a 2-m propane bubble chamber at the JINR was unexpected. The chamber was irradiated with $10-\mathrm{GeV}$ protons and the particle energy and momentum were determined from the dynamics of changes in the track curvature in a magnetic field. These particles were called anomalous leptons, although the particle spin was not determined in the experiment [1].
An experimental setup (Fig. 1) was created to perform a new search for anomalous leptons in the bremsstrahlung photon beam with energies of up to $500 \mathrm{MeV}$ at the Pakhra accelerator of the LPI. The time-offlight technique is used to determine the momentum and energy of particles escaping from the target. The Start signal is produced by a system of trigger scintillation counters $S_{1}-S_{3}$ located behind the target $T$. The Stop signal is generated by the signal from the counter $S_{4}$ located in front of the scintillation spectrometer (SS), which detects particles produced by bremsstrahlung photons in the target and has dimensions of $200 \times$ $200 \times 200 \mathrm{~mm}$.

The maximum electron energy that the SS is capable of detecting is approximately $E \approx 40 \mathrm{MeV}(E \approx$ $(\Delta E / \Delta x) L$, where $\Delta E / \Delta x$ is the mean energy loss of electrons per unit track length in the detector and $L$ is the scintillation detector thickness $[2,3])$. The

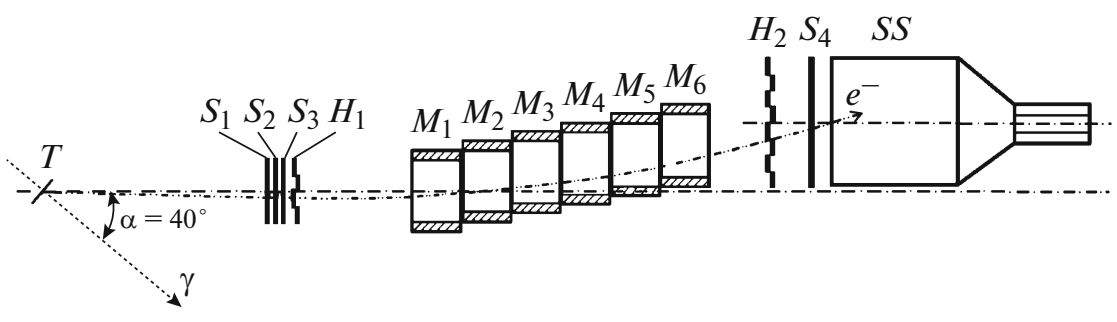

Fig. 1. The diagram of the experimental setup for searching for a heavy lepton at the LPI Pakhra accelerator: $(T)$ copper target, $\left(S_{1}-S_{4}\right)$ scintillation counters, $\left(H_{1}, H_{2}\right)$ scintillation hodoscopes, $\left(M_{1}-M_{6}\right)$ sections of permanent magnets, and (SS) scintillation spectrometer. 


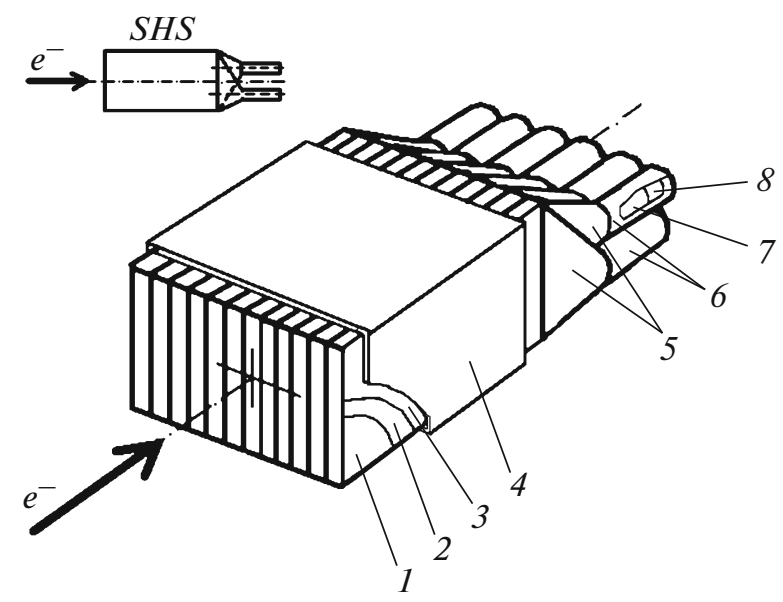

Fig. 2. The diagram of the SHS scintillation hodoscopic spectrometer: (1) scintillation plate, (2) metallized Mylar, (3) black paper, (4) metal case, (5) light collectors, (6) case of (7) FEU-85 PMT with (8) voltage divider, and (SHS) scintillation hodoscopic spectrometer (in the inset).

momentum of a particle escaping from the target is determined from the deflection angle of the particle in the system of permanent magnets $M_{1}-M_{6}$ that are located on its trajectory. The particle trajectory is determined by the exit point of the particle from the target, which is assumed to be the center of the target (for definiteness), and by the triggered channels of the hodoscopes $H_{1}$ and $H_{2}$, which contain four and eight channels, respectively. The channels of both hodoscopes have equal dimensions, which are $50 \times 20 \times 5$ $\mathrm{mm}$. The hodoscope $H_{1}$ is located behind the trigger counters $S_{1}-S_{3}$, and $H_{2}$ is located in front of the counter $S_{4}$.

\section{THE SCINTILLATION HODOSCOPIC SPECTROMETER}

To improve the accuracy in determining the momentum of a detected particle, which is specified by on the deflection angle of the particle in the magnetic field produced by the magnet system, we decided to transfer the hodoscope $H_{2}$ directly to the magnets, remove the counter $S_{4}$ from the setup, and replace the SS with a hodoscopic spectrometer, which should simultaneously measure the particle energy and coordinate. The scintillation hodoscopic spectrometer (SHS, see Fig. 2) has been created for this purpose.

The SHS is an assembly of 12 independent channels, i.e., polystyrene-based scintillator plates 1 with dimensions of $500 \times 250 \times 20 \mathrm{~mm}$. Each plate is viewed from its end face through the air light collector 5 by a single FEU-85 photomultiplier tube (PMT) 7. The height of each light collector from the end plane of the scintillator plate to the PMT photocathode is $15 \mathrm{~cm}$. Since the plate width is $20 \mathrm{~mm}$ and the FEU- 85 bulb diameter is

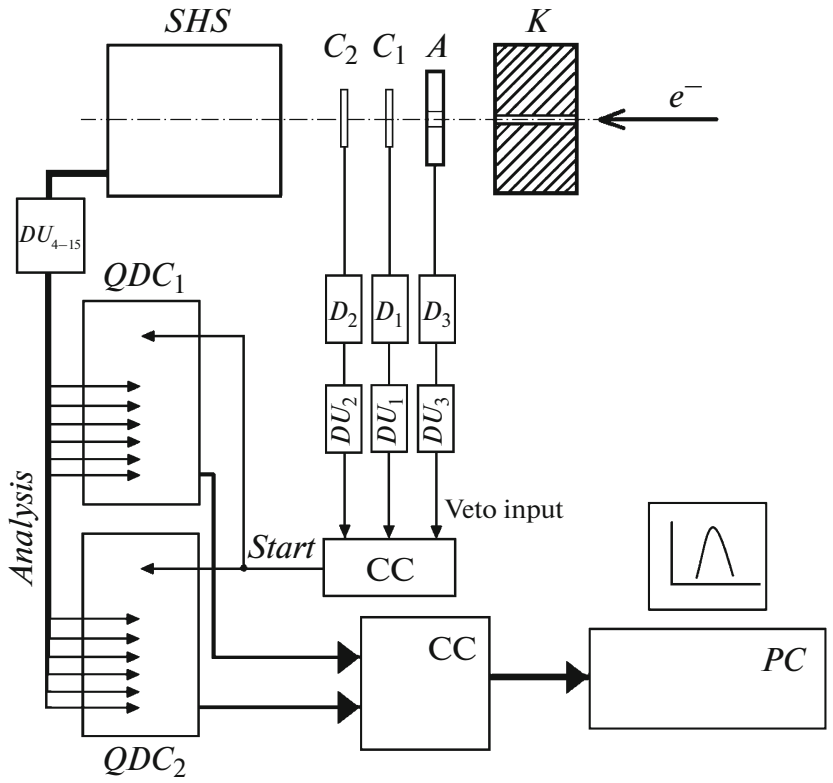

Fig. 3. The block diagram of the calibration channel of quasi-monochromatic secondary electrons at the LPI Pakhra accelerator: $\left(D_{1}-D_{3}\right)$ discriminator units, $\left(D U_{1}-\right.$ $\left.D U_{15}\right)$ delay units, (CC) coincidence circuit, $\left(Q D C_{1}\right.$, $Q D C_{2}$ ) 8-input charge-to-digital converters, (Start) trigger signal, (Analysis) amplitude signals from SHS channels, $(C C)$ CAMAC system crate controller, and $(P C)$ personal computer.

$30 \mathrm{~mm}$, the PMT symmetry axis is shifted in the vertical plane by $40 \mathrm{~mm}$ relative to the symmetry axis of the scintillator plate (see the inset in Fig. 2) for compactness. In the working position, the odd-numbered light collectors and PMTs are located vertically one after another above the symmetry axis of the plate, and the even-numbered light collectors and PMTs are located below.

All edges of the plate, except for the plate on which a light collector with a PMT is located, are coated with metalized Mylar 2 and black paper 3. The assembly is placed in a metal case 4 to strengthen the structure, but the end part of the assembly on which particles should be incident and the rear part of the assembly on which light collectors with the PMT are located are not covered by the case.

\section{CALIBRATION OF THE SCINTILLATION HODOSCOPIC SPECTROMETER}

The SHS was calibrated using a quasi-monochromatic secondary-electron beam of the LPI Pakhra accelerator $[4,5]$. The block diagram of the calibration channel is shown in Fig. 3. The signals from the trigger counters $C_{1}$ and $C_{2}$ with dimensions of $10 \times 10 \times 5 \mathrm{~mm}$ were fed via the discriminator units $D_{1}$ and $D_{2}$ and delay units $D U_{1}$ and $D U_{2}$ to the inputs of the coincidence circuit (CC). The signal from anticoincidence counter $A$ with dimensions of $90 \times 60 \times 10 \mathrm{~mm}$ with a 
10-mm-diameter hole was fed via the discriminator unit $D 3$ and delay unit $D U 3$ to the Veto input of the CC.

The signal from the CC was the Start trigger signal for triggering two units of eight-input charge-to-digital converters (QDCs). The signals from 12 SHS channels were fed to the Analysis inputs of these QDCs through the delay units $D U_{4}-D U_{15}$. The Start signal was also used as a signal that initiated recording of the SHS signals to the computer memory through the CAMAC crate controller. In front of the anticoincidence counter $A$ was a lead collimator with a 3 -mm-diameter hole, which specified the electron-beam aperture. The intensity of the electron beam was $\sim 15 e^{-} / \mathrm{s}$.

The SHS was calibrated in two stages. At the first stage, the signal amplitudes of all SHS channels on the secondary electron beam were sequentially equalized so that the amplitude spectrum of each channel fitted to the QDC operating region. The length of each SHS plate is $50 \mathrm{~cm}$, so the mean energy loss of electrons in the scintillator is $\langle E\rangle \approx 2[\mathrm{MeV} / \mathrm{cm}] \times 50[\mathrm{~cm}] \approx 100 \mathrm{MeV}$. Since the energy deposited in the SHS does not increase at electron energies above $100 \mathrm{MeV}$ [3], the maximum energy loss in the SHS channel should also be no greater than $\langle E\rangle=\left\langle E_{\max }\right\rangle \approx 100 \mathrm{MeV}$. Therefore, the first stage of calibration was carried out at the electron-beam energy $E=80 \mathrm{MeV}$.

As a result, the average amplitude of each channel corresponded to $\sim 260$ QDC channels with a maximum operating range of 512 channels. The mean amplitude of the SHS channel was changed by varying the voltage across the PMT voltage divider in this channel. The positions of channels relative to the electron beam in the horizontal plane were remotely changed with an accuracy of $\sim 1 \mathrm{~mm}$ by moving the platform with the SHS fixed in place on it.

\section{THE ENERGY RESOLUTION OF THE SHS}

The energy calibration of the SHS was performed at the second stage. The spectrometer was placed on the electron beam in such a position that electrons hit the center of the seventh channel at an angle of $0^{\circ}$ relative to the beam trajectory and the axis of symmetry of the channel plate.

The SHS energy calibration was performed at electron energies of $E=40,60,80,100,120,150$, and $200 \mathrm{MeV}$. The amplitude of the SHS that detected the $i$ th electron was determined by the sum of the amplitudes in all channels $A_{i}=\sum_{m=1}^{12} A_{m i}$, where $A_{m i}$ is the amplitude in the $m$ th SHS channel, and $m=1, \ldots, 12$ is the channel number.

The electron energy was evaluated as $E_{i}=k A_{i}$, where $k$ is the proportionality factor and $A_{i}$ is the amplitude of the SHS that detected the $i$ th electron. The factor $k$ was determined from the SHS calibration on the electron beam with energy $E=60 \mathrm{MeV}$ and was then verified and corrected on the electron beam with energy $E=40 \mathrm{MeV}$
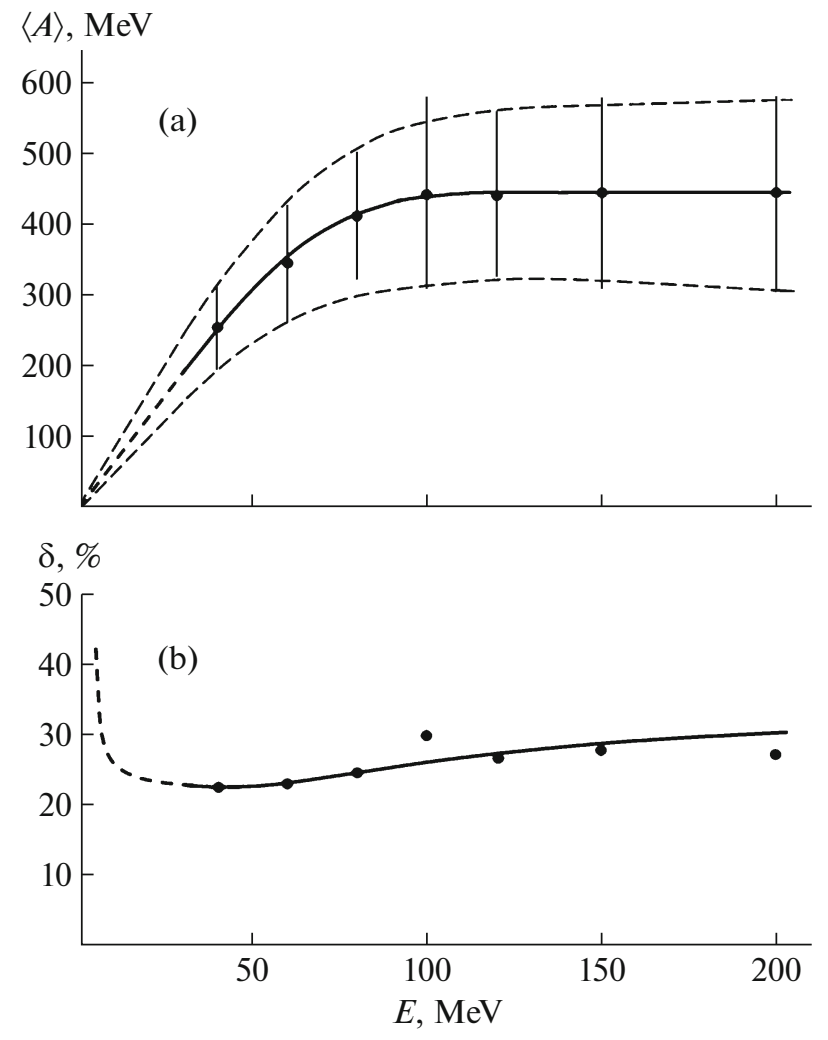

Fig. 4. The dependences of SHS characteristics on the electron energy: (a) mean amplitude and (b) relative energy resolution of the SHS.

( $k=E / \sum_{m=1}^{12}\left\langle A_{m}\right\rangle$, where $E$ is the energy of the calibrating electron beam and $\left\langle A_{m}\right\rangle$ is the mean amplitude of the spectrum in the $m$ th channel $(m=1, \ldots, 12)$ determined from the calibration dataset).

The energy dependences of the mean SHS amplitude and relative amplitude resolution are shown in Fig. 4. The dependence in Fig. 4a qualitatively reproduces the dependence of the change in the amplitude of the $20-\mathrm{cm}$-thick scintillation spectrometer on the energy of the secondary electron beam presented in [3]. It can be seen that the energy loss in SHS increases with the electron energy up to $100 \mathrm{MeV}$ and is constant at electron energies from $100 \mathrm{MeV}$ to the last studied energy of $200 \mathrm{MeV}$. This means that up to an electron energy of $100 \mathrm{MeV}$ the energy loss of electrons completely "fits" into the SHS volume that is determined by the spectrometer length along the beam trajectory. From Fig. 4a, it can also be seen that the dependence of the mean SHS amplitude on the electron energy is linear to $\sim 70 \mathrm{MeV}$, is nonlinear with a transient character in the region from $\sim 70$ to $\sim 100 \mathrm{MeV}$, and is determined by a constant value at energies above $\sim 100 \mathrm{MeV}$.

The relative amplitude resolution of the SHS, which also acts as the relative energy resolution, was defined as $\delta=((\Delta A /\langle A\rangle) / 2.35) \times 100 \%$, where $\Delta A$ is the full width at half maximum of the amplitude spec- 


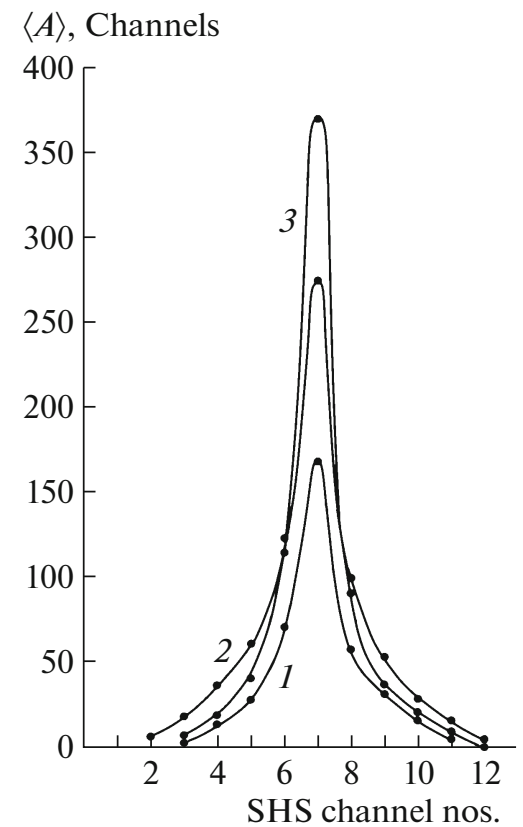

Fig. 5. The longitudinal profiles of the development of electromagnetic showers produced by electrons in the SHS, as functions of the energy: $E=$ (1) 40 , (2) 100 , and (3) $200 \mathrm{MeV}$.

trum, $\langle A\rangle$ is the mean amplitude of the SHS amplitude spectrum, and $2.35=2(\ln 2) \sqrt{2}$. Figure $4 \mathrm{~b}$ shows that the dependence of the SHS amplitude resolution on the electron energy is complex. If we extrapolate the experimental errors towards the region of low electron energies (Fig. 4a), we can see (Fig. 4b) that $\delta$ improves significantly as the electron energy increases from $5 \mathrm{MeV}(\delta \sim$ $40-50 \%$ ) up to an energy of $\sim 40 \mathrm{MeV}$, at which the SHS has the best resolution $\delta \approx 22 \%$. Further increase in the electron energy leads to deterioration of $\delta$ and, starting with an electron energy of $100 \mathrm{MeV}$, the dependence becomes almost linear and reaches the value $\delta \approx 31 \%$ at an electron energy of $200 \mathrm{MeV}$.

\section{THE TRANSVERSE PROFILES OF ELECTROMAGNETIC SHOWERS}

Figure 5 shows profiles of electromagnetic showers that developed in the SHS in the transverse direction at electron-beam energies $E=40,100$, and $200 \mathrm{MeV}$. It is seen that the energy loss in all SHS channels increases with an increase in the electron energy; however, the energy loss in the peripheral channels decreases starting with $E>100 \mathrm{MeV}$.

Figure 6 shows the energy dependence of the change in the transverse width of an electromagnetic shower in the SHS. At electron energies of up to $E \approx$ $100 \mathrm{MeV}$, the shower width can be estimated as $\Delta \approx$ $\alpha \ln \beta E$, where $\Delta$ is the width of the electromagnetic shower in terms of SHS channels, $E[\mathrm{MeV}]$ is the electron energy, $\alpha=2.183$, and $\beta=1.544 \mathrm{MeV}^{-1}$ [2]. The shower

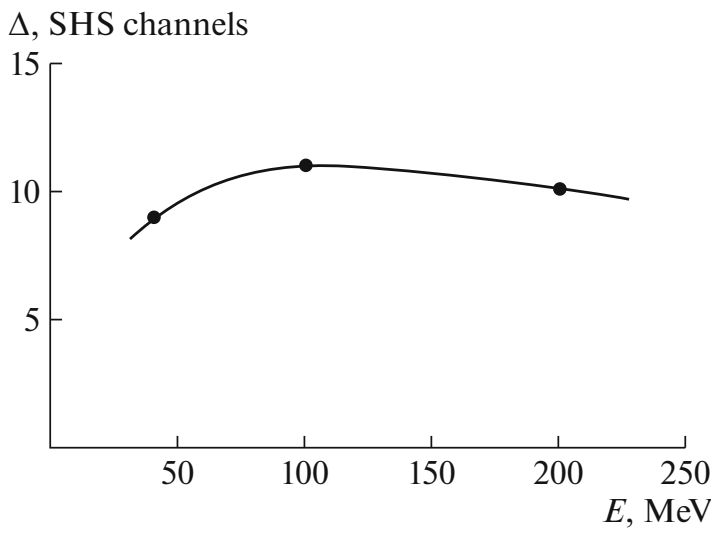

Fig. 6. The width of electromagnetic showers produced by electrons in the SHS, as functions of the electron energy: $(\Delta)$ width of an electromagnetic shower in terms of the SHS channels.

width becomes smaller at energies $E>100 \mathrm{MeV}$ and the dependence can be described by the estimate $\Delta \approx$ $\alpha \exp (-\beta E)$ at $\alpha=12.1$ and $\beta=-9.531 \times 10^{-4} \mathrm{MeV}^{-1}$. It can be assumed that a decrease in the width of an electromagnetic shower with an increase in the electron energy is associated with an increase in the mean energy of secondary charged particles of the shower $\left(e^{-} e^{+}\right.$pairs) and, therefore, with a decrease in the mean angle of multiple scattering [6].

\section{THE COORDINATE RESOLUTION OF THE SHS}

The coordinates of the hit point of electrons at the SHS was determined using the center-of-gravity method [6]. The coordinate of the $i$ th electron was $x_{i}=$ $d\left(\sum_{m=1}^{12} m A_{m i} / \sum_{m=1}^{12} A_{m i}\right)$, where $d$ is the SHS channel width, $A_{m i}$ is the signal amplitude in SHS channel $m$, and $m$ is the channel number $(m=1, \ldots, 12)$. The coordinate determined by this method has an offset $x_{0}$. The measurements showed that the offset is energy-independent in the range of the studied energies from 40 to $200 \mathrm{MeV}$ and its value is $x_{0}=52.5 \mathrm{~mm}$. Figure 7 shows the dependence of the SHS coordinate resolution $\sigma_{x}$ on the electron energy in view of the subtraction of the electron beam width, which is determined by the collimator diameter $(3 \mathrm{~mm})$ in front of the trigger counters $C_{1}$ and $C_{2}$ (Fig. 3).

From Fig. 7, one can see that the dependence of $\sigma_{x}$ on the electron energy with a small numerical shift in the energy is similar in character to the dependence of the relative energy resolution $\delta$. The energy dependence of $\sigma_{x}$ is linear at electron energies up to $\sim 80 \mathrm{MeV}$ and nonlinear in the range from $\sim 80$ to $\sim 120 \mathrm{MeV}$ and a linear decrease is observed at electron energies above $\sim 120 \mathrm{MeV}$. 


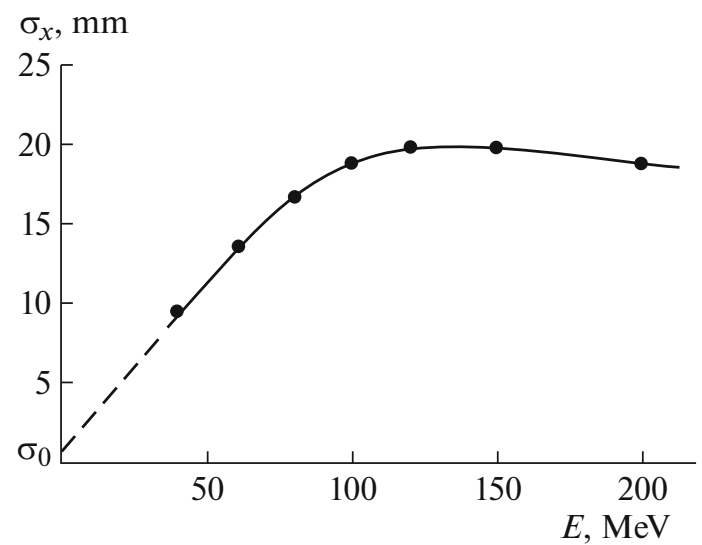

Fig. 7. The dependence of the SHS coordinate resolution on the electron energy.

Figure 8 shows the energy dependence of the change in the width of the electromagnetic shower initiated in xenon by a $\gamma$-ray photon [7]. It is seen that the behavior of the dependence in Fig. 7 qualitatively corresponds to the behavior of the dependence in Fig. 8 . Thus, at the studied electron energies and a constant SHS channel width, the dependence of $\sigma_{x}$ is determined by the energy dependence of the change in the width of an electromagnetic shower.

The energy dependence of $\sigma_{x}$ is linear at electron energies $E$ below $\sim 80 \mathrm{MeV}$. It can be described by the expression $\sigma_{x}=\sigma_{x}(E)=\sigma_{0}+a E$ at $\sigma_{0}=0.5 \mathrm{~mm}$ and $a=0.225 \mathrm{~mm} / \mathrm{MeV}$. Beginning with energies $E \sim 120 \mathrm{MeV}$ and higher, the shower width depends on the energy to a lesser degree (Fig. 8) and therefore has a weaker effect on $\sigma_{x}$, which obeys the dependence $\sigma_{x}(E) \sim E^{-1 / 2}$ in this energy range $[6,8]$. The dependence manifests itself when the spectrometer thickness in the longitudinal direction is $t_{\text {spectr }}>2 \mathrm{X}_{0}\left(\mathrm{X}_{0}\right.$ is the radiation length), since the maximum shower development and, therefore, the maximum shower width in the transverse direction (curve 1 in Fig. 8), correspond to this thickness beginning with an energy of $\sim 120 \mathrm{MeV}$.

The SHS thickness is $1.25 \mathrm{X}_{0}$, and the shower development maximum falls beyond the SHS volume at electron energies greater than $\sim 120 \mathrm{MeV}$. In this case, the shower width is determined by the point of the shower development to its maximum and this width is slightly less than the shower width at the maximum. Therefore, $\sigma_{x}$ also decreases, obeying the linear law (curve 2 in Fig. 8). The energy range $\sim 80<E<\sim 120 \mathrm{MeV}$ is the intermediate region of $\sigma_{x}$ variation.

The $\sigma_{x}$ value varies from $\sigma_{x}=9.5 \mathrm{~mm}$ at $E=40 \mathrm{MeV}$ to $\sigma_{x} \approx 20 \mathrm{~mm}$ at $E=120 \mathrm{MeV}$. The coordinate resolution of the SHS at $E=200 \mathrm{MeV}$ is $\sigma_{x} \approx 19 \mathrm{~mm}$.

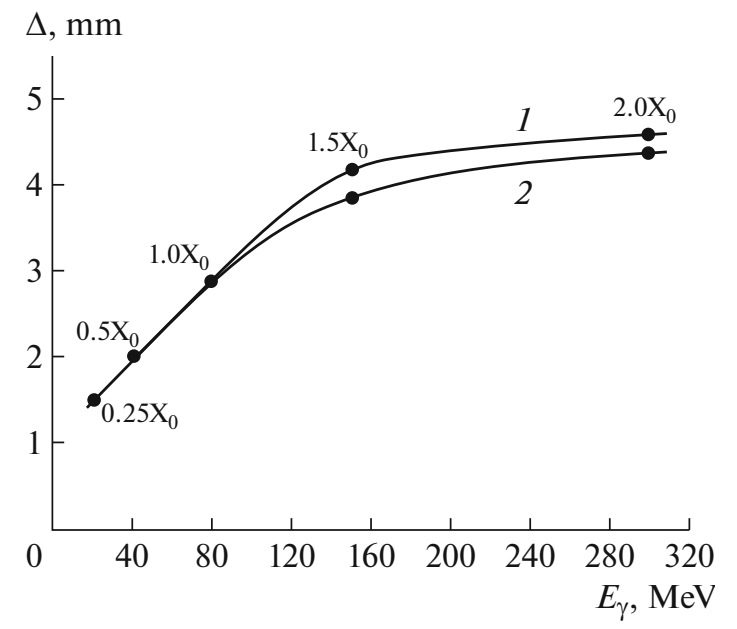

Fig. 8. The energy dependence of the width of an electromagnetic shower produced by $\gamma$ rays in xenon: (1) shower width at the maximum of the shower development (the shower depths $t$ at which the maximum is located are designated by numbers) and (2) shower width at the depth of the shower development $t \approx 1.3 \mathrm{X}_{0}$.

\section{THE DEPENDENCE OF THE SHS CHARACTERISTICS ON THE ANGLE OF INCIDENCE OF ELECTRONS ON THE SPECTROMETER}

The dependence of the SHS characteristics on the angle of incidence $\Theta$ of electrons on the spectrometer with respect to their trajectory in the horizontal plane were investigated using an electron beam with energy $E=80 \mathrm{MeV}$. Figure 9 presents the dependences of the mean amplitude, the relative amplitude resolution, and the coordinate resolutions, as well as the electron coordinate determined by the SHS, on the angle of incidence of electrons on the spectrometer. In Fig. 9a, it can be seen that the mean amplitude of the SHS signals is constant in the angular range of $0^{\circ}-1.5^{\circ}$ and begins to increase at an angle of $\sim 2^{\circ}$. The increase in the amplitude at the maximum studied angle $\Theta=5.7^{\circ}$ relative to the amplitude at an angle of $0^{\circ}$ is as high as $\sim 22 \%$. Figure $9 \mathrm{~b}$ shows that the relative amplitude resolution $\delta$ and the coordinate resolution $\sigma_{x}$ also increase with an increase in the angle of incidence of electrons on the SHS. Starting from angles of about $1.5^{\circ}-2.0^{\circ}$ the increase in the $\delta$ and $\sigma_{x}$ values is greater than $30 \%$. Figure $9 \mathrm{c}$ demonstrates the linear dependence of the offset of the coordinate determined by the SHS on the angle of incidence of an electron on the spectrometer. This offset can be corrected by the dependence $x=a+$ $b \Theta$ at $a=0.03 \mathrm{~mm}$ and $b=0.771 \mathrm{~mm} / \mathrm{deg}$.

\section{CONCLUSIONS}

The calibration of the scintillation hodoscopic spectrometer on the secondary electron beam of the Pakhra accelerator with energies of $40-200 \mathrm{MeV}$ 

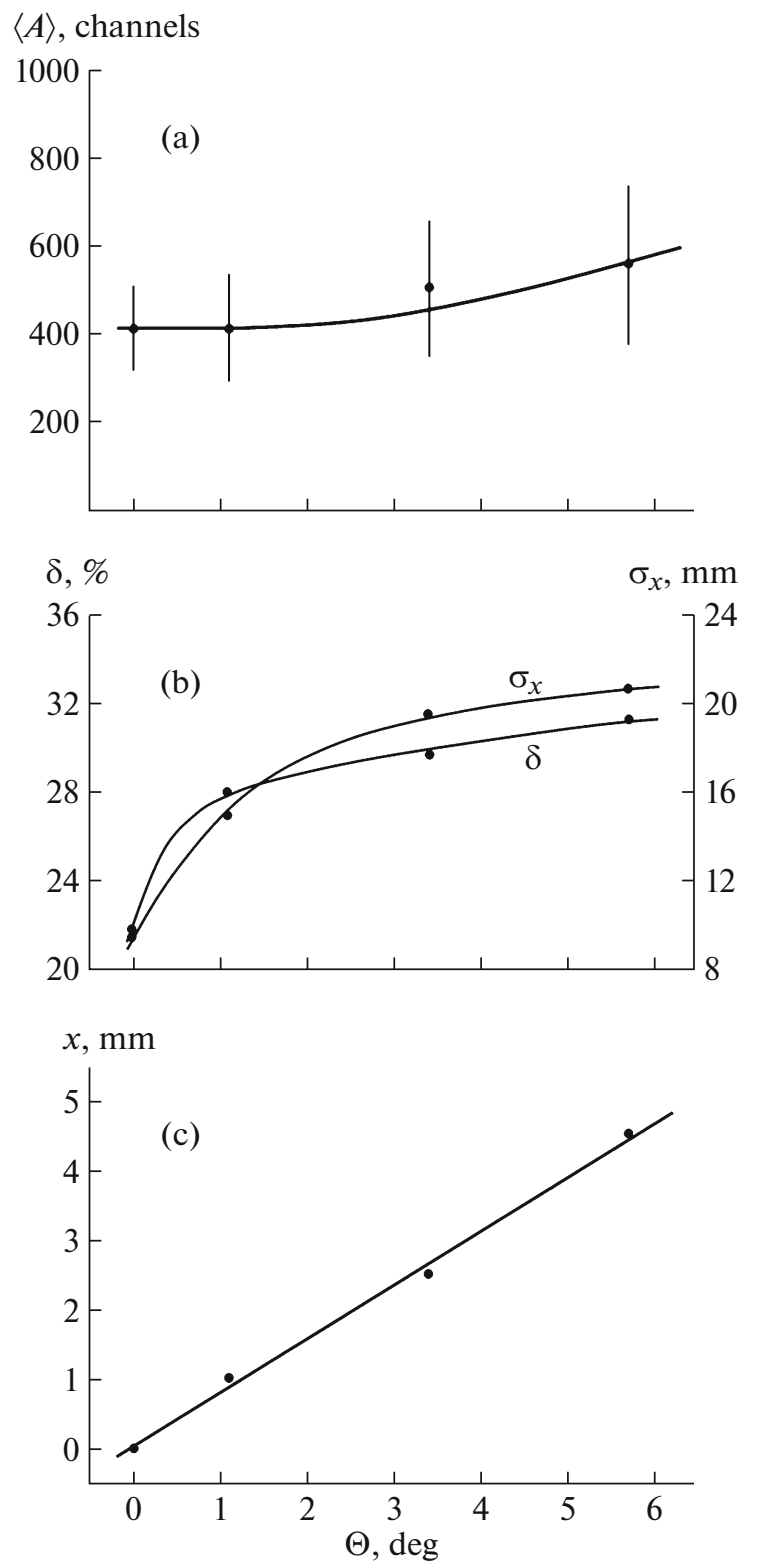

Fig. 9. The dependence of the energy and coordinate characteristics of the SHS on the angle of incidence of electrons onto the SHS at electron energy $E=80 \mathrm{MeV}$ : (a) mean amplitude of the SHS, (b) relative energy $\delta$ and coordinate $\sigma_{x}$ resolution of SHS, and (c) coordinate of the hit point of electrons at the SHS.

shows that the energy dependence of the mean amplitude obeys a linear law up to $\sim 70 \mathrm{MeV}$ in the range of electron energies $E<100 \mathrm{MeV}$ at which the search experiment is carried out. The relative amplitude resolution and, therefore, the energy resolution of the SHS are minimal at electron energy $E=40 \mathrm{MeV}$ and change only slightly with an increase in the electron energy to $\sim 100 \mathrm{MeV}$, being at a level of $\delta=22-25 \%$. The coordinate resolution of the SHS varies linearly from $\sigma_{x}=9.5 \mathrm{~mm}$ at $E=40 \mathrm{MeV}$ to $\sigma_{x}=17 \mathrm{~mm}$ at $E=$ $80 \mathrm{MeV}$.
It has been determined that the transverse shower width $\Delta$ obeys the dependence $\Delta \sim \ln E$ at electron energy $E<100 \mathrm{MeV}$. It has been ascertained that the mean SHS amplitude, the amplitude and coordinate resolutions, and the coordinate determined by the SHS depend on the angle of incidence of electrons on the SHS. A simple correcting function $x=a+b \Theta$ can be used to determine the exact coordinates of the hit point of electrons at the SHS. Additional studies will be carried out to determine the dependence of the coordinate offset on the angle of incidence of electrons onto the SHS and on the electron energy.

The SHS is capable of detecting charged particles with energies in the range of up to $\sim 80 \mathrm{MeV}$, which is two times larger than the detection range of the currently used scintillation spectrometer and better with an accuracy of $\approx 20 \%$ relative to the spectrometer of the same type in [3]. The SHS is capable of determining the coordinate of a particle hit point at the spectrometer with an accuracy no worse than the accuracy of the scintillation hodoscope that is now located in front of the SS. The scintillation hodoscopic spectrometer can be used in an experiment to search for anomalous leptons.

\section{REFERENCES}

1. Alekseev, V.I., Baskov, V.A., Dronov, V.A., Krechetov, Yu.F., L'vov, A.I., Pavlyuchenko, L.N., Polyanskiy, V.V., and Sidorin, S.S., EPJ Web Conf., 2019, vol. 204, p. 08009. https://doi.org/10.1051/epjconf/201920408009.

2. Kalinovskii, A.N., Mokhov, N.V., and Nikitin, Yu.P., Prokhozhdenie chastits vysokikh energii cherez veshchestvo (Passage of High-Energy Particles Through Matter), Moscow: Energoatomizdat, 1985.

3. Alekseev, V.I., Baskov, V.A., Dronov, V.A., L'vov, A.I., Koltsov, A.V., Krechetov, Yu.F., and Polyansky, V.V., 2020. arXiv:2004.00937.

4. Alekseev, V.I., Baskov, V.A., Dronov, V.A., L'vov, A.I., Koltsov, A.V., Krechetov, Yu.F., Polyansky, V.V., and Sidorin, S.S., 2020. arXiv:1912.08095.

5. Alekseev, V.I., Baskov, V.A., Dronov, V.A., L'vov, A.I., Krechetov, Yu.F., Malinovsky, E.I., Pavlyuchenko, L.N., Polyansky, V.V., and Sidorin, S.S., Instrum. Exp. Tech., 2019, vol. 62, no. 2, pp. 143-149. https://doi.org/10.1134/S0020441219020143

6. Belousov, A.S., Vazdik, Ya.A., Zverev, V.G., Malinovskii, E.I., Malinovskii, I.E., and Terkulov, A.R., Preprint of Lebedev Physical Institute Russ. Acad. Sci., Moscow, 1995, no. 23.

7. Ogrzeval'skii, Z. and Strugal'skii, Z.S., Preprint of Joint Institute for Nuclear Research, Dubna, 1969, no. R14562.

8. Binon, F., Buyanov, V.M., Guaner, M., Donskov, S.V., Dytel, P., Dyfurno, G., Inyakin, A.V., Kakauridze, D.B., Kachanov, V.A., Kulik, A.V., Lanie, G.P., Lednev, A.A., Mikhailov, Yu.V., Mishot, D., Muti, T., et al., Preprint of Institute for High Energy Physics, Serpukhov, 1985, no. 85-62.

Translated by N. Goryacheva 